Wright State University

CORE Scholar

Computer Science and Engineering Faculty

Publications

Computer Science \& Engineering

7-1-2007

\title{
Quo Vadis, CS? - On the (Non)-Impact of Conceptual Structures on the Semantic Web
}

\author{
Sebastian Rudolph \\ Markus Krotzsch \\ Pascal Hitzler \\ pascal.hitzler@wright.edu
}

Follow this and additional works at: https://corescholar.libraries.wright.edu/cse

Part of the Bioinformatics Commons, Communication Technology and New Media Commons, Databases and Information Systems Commons, OS and Networks Commons, and the Science and Technology Studies Commons

\section{Repository Citation}

Rudolph, S., Krotzsch, M., \& Hitzler, P. (2007). Quo Vadis, CS? - On the (Non)-Impact of Conceptual Structures on the Semantic Web. Lecture Notes in Computer Science, 4604, 464-467.

https://corescholar.libraries.wright.edu/cse/135

This Conference Proceeding is brought to you for free and open access by Wright State University's CORE Scholar. It has been accepted for inclusion in Computer Science and Engineering Faculty Publications by an authorized administrator of CORE Scholar. For more information, please contact library-corescholar@wright.edu. 


\title{
Quo Vadis, CS? - On the (non)-impact of Conceptual Structures on the Semantic Web (Position Paper)
}

\author{
Sebastian Rudolph, Markus Krötzsch, and Pascal Hitzler \\ Institute AIFB, Universität Karlsruhe (TH), Germany \\ \{rudolph, kroetzsch, hitzler\}@aifb.uni-karlsruhe.de
}

\begin{abstract}
Conceptual Structures is a field of research which shares abstract concepts and interests with recent work on knowledge representation for the Semantic Web. However, while the latter is an area of research and development which is rapidly expanding in recent years, the former fails to participate in these developments on a large scale. In this paper, we attempt to stimulate the Conceptual Structures community to catch the Semantic Web train.
\end{abstract}

\section{Status Quo}

It is a fact that mainstream Semantic Web (SW) developments currently happen with only little impact from the Conceptual Structures (CS) community. This is a curious development as Semantic Web knowledge representation is closely related to CS research. While CS certainly profits from the SW hype - as do many areas in computer science - we believe that the full potential for the transfer and use of CS methods and technologies is not given enough credit by the community.

With this somewhat provocative position paper, we intend to stimulate a controversial discussion about the possible future of Conceptual Structures as the changed situation might require to reconsider former pessimistic attitudes towards the Semantic Web as depicted in [1]. We first give a very brief history of Conceptual Structures and Semantic Web. We then analyse the two main paradigms for the CS community, namely Conceptual Graphs (CGs) and Formal Concept Analysis (FCA) in terms of their relationship to SW research. For each of the paradigms, we will give general research directions which we think would help to leverage CS for the SW.

We will substantiate some of our statements and claims by literature references, but there is too much work which is important for this position paper to explicitly refer to it all. The resulting selection is obviously very subjective.

Conceptual Structures is a term introduced by John Sowa in his 1984 book on the topic [2]. His work stimulated an interdisciplinary research community with interests in the relations between artificial intelligent knowledge representation, mathematical logic, philosophy and linguistics, manifesting itself in the annual International Conference on Conceptual Structures ${ }^{1}$ which runs since 1993 after some workshops in the years before.

\footnotetext{
${ }^{1}$ See http://www . conceptualstructures .org.
} 
The field basically comprises two interacting research communities, one of which focuses on conceptual graphs as introduced in [2], while the other comes from a tradition spawned by Rudolf Wille's work in 1982 on restructuring lattice theory [3], which led to the establishment of Formal Concept Analysis [4] as a mathematical theory and which recently finds applications in Computer Science, especially in data mining [5].

While the CS community mainly focusses on the above-mentioned areas, it also has strong mathematical and philosophical undercurrents, and also ever since has been involved in Computer Science applications, witnessed by a considerable number of implemented systems and application studies.

Semantic Web, in contrast, is a relatively new research area spawned by Tim BernersLee, inventor of the World Wide Web and director of the World Wide Web Consortium (W3C) [6]. Its main idea is to bring meaning to web data for intelligent processing. This is achieved by utilising knowledge representation languages for describing socalled ontologies, which model domains of interest in a logic-based, declarative and machine-processable way. Ontology representation languages which have been standardised by the W3C are the Resource Description Framework RDF(S) ${ }^{2}$ and the Web Ontology Language $\mathrm{OWL}^{3}$, both of which have concept hierarchies as their basic internal structure. While RDFS is a straightforward language which features only a simple use of inheritance for inferencing, OWL is a full-blown Description Logic (DL) [7], and as such a powerful knowledge representation language.

Semantic Web research in the last few years was driven by the W3C and by influential funding agencies such as DARPA and the European Commission. Specialised annual conferences, like the International Semantic Web Conference feature several hundred participants each year, and Semantic Web publications are present in all major conferences and journals in Artificial Intelligence, Knowledge Management, and other fields. Influential software companies like IBM, Oracle and SAP are currently starting to enter the market with products based on the underlying Semantic Technologies.

\section{Quo Vadis, CG?}

Both being historically founded in semantic networks, CGs and DLs share a conceptually very similar view on how to represent knowledge, as reported by Tim Berners-Lee. ${ }^{4}$ It is thus surprising that the participation of the $\mathrm{CG}$ community in mainstream Semantic Web research is very limited.

While CGs have been used in some Semantic Web applications (see e.g. the Corese Semantic Web Factory ${ }^{5}$ or [8]), such work is rarely presented at mainstream Semantic Web events and has had next to no impact on standardisation efforts. So, while CGs are the more historic approach, DLs overtook and got standardised. Considering the high impact in research and development of SW methods and technologies, it is promising to utilise the close conceptual relationship between CGs and SW languages, and to utilise

\footnotetext{
${ }^{2}$ http://www .w3 .org/RDF/

${ }^{3}$ http://www.w3.org/2004/0wL/

${ }^{4}$ http://www.w3.org/DesignIssues/CG.html

${ }^{5}$ http://www-sop.inria.fr/acacia/corese/
} 
CGs for the Semantic Web. In detail, we identify the following issues which seem to be particularly interesting for being taken up immediately.

- Thoroughly investigate the relationships between CGs and standardised or widely used ontology languages in order to mediate a knowledge transfer from CGs to SW languages and technologies [9].

- Employ CG technology for the visualisation of inferencing in order to explain to the naive user how implicit knowledge is being derived.

- Leverage CG technology for building visual ontology user interfaces that can be used by non-experts.

\section{Quo Vadis, FCA?}

FCA is very limited as a knowledge representation formalism, as it is basically restricted to concept hierarchies, with some minimal logical flavour [10,11]. Taxonomies and hierarchies, however, are fundamental to Semantic Web knowledge representation, as witnessed e.g. by the $\mathrm{RDF}(\mathrm{S})$ standard and also the fact that practical ontology modelling is usually done by initially creating concept hierarchies. Because of this, FCA can be utilised as a data mining tool for creating drafts for basic ontologies by automated means, which can subsequently be extended - see e.g. [12] or [13]. As such, FCA has indeed been established in the Semantic Web to a certain extent.

But the natural question arises, how the impact of FCA can be further pushed in order to become a more prominent basic technology for the Semantic Web. We see the following promising possible lines of development.

- Investigate expressive knowledge representation formalisms such as DLs and their extensions in their relation to FCA $[14,15]$ in order to leverage FCA for more expressive formalisms by overcoming the fixation on concept hierarchies.

- Further investigate FCA approaches towards ontology creation and refinement [16].

- Investigate the use of FCA for user interaction with ontologies [17].

\section{Quod Differtur, Non Aufertur}

The CS community can draw on a rich history and well-developed methods on its path into the future. We believe that it has the potential to leverage substantial impact on the current Semantic Web trend. In order to do this, however, efforts are needed along the following lines.

- CS paradigms need to be studied in depth in their comparison with SW knowledge representation paradigms.

- CS needs to reflect about its own strengths in relation to concrete needs in Semantic Web research.

- Concerted efforts have to be undertaken to disseminate CS methods and established knowledge in the SW community.

Finally, it must not be forgotten that the Semantic Web - an extension of the current Web - also is an applied area of research, where well-specified file formats, stable software tools, standardised data types, Unicode and URIs are as important as wellfounded representation formalisms. 
Acknowledgements. This work is supported by the German Federal Ministry of Education and Research (BMBF) under the SmartWeb project (grant 01 IMD01 B), and by the Deutsche Forschungsgemeinschaft (DFG) under the ReaSem project.

\section{References}

1. Priss, U.: Alternatives to the "semantic web": multi strategy knowledge representation. In: Proc. ISKO 2002. (2002)

2. Sowa, J., ed.: Conceptual Structures: Information Processing in Mind and Machine. Addison-Wesley, Reading, MA (1984)

3. Wille, R.: Restructuring lattice theory: An approach based on hierarchies of concepts. In Rival, I., ed.: Ordered Sets. Reidel, Dordrecht-Boston (1982) 445-470

4. Ganter, B., Wille, R.: Formal Concept Analysis - Mathematical Foundations. Springer, Berlin (1999)

5. Stumme, G.: Formal concept analysis on its way from mathematics to computer science. In Priss, U., Corbett, D., (eds.), G.A., eds.: Conceptual Structures: Integration and Interfaces, Proc. ICCS 2002. LNAI, Springer (2002) 2-19

6. Berners-Lee, T., Hendler, J., Lassila, O.: The semantic web. Scientific American (2001)

7. Baader, F., Calvanese, D., McGuinness, D., Nardi, D., Patel-Schneider, P., eds.: The Description Logic Handbook: Theory, Implementation, and Applications. Cambridge University Press (2003)

8. Dieng-Kuntz, R., Corby, O.: Conceptual graphs for Semantic Web applications. In Dau, F., Mugnier, M.L., Stumme, G., eds.: Proc. ICCS 2005. Volume 3596 of LNCS., Springer (2005) 19-50

9. Baader, F., Molitor, R., Tobies, S.: Tractable and decidable fragments of conceptual graphs. In: Proc. ICCS 1999, London, UK, Springer (1999) 480-493

10. Ganter, B., Wille, R.: Contextual attribute logic. In Tepfenhart, W.M., Cyre, W.R., eds.: Proc. ICCS 1999. Volume 1640 of LNAI., Springer (1999) 377-388

11. Hitzler, P., Krötzsch, M., Zhang, G.Q.: A categorical view on algebraic lattices in formal concept analysis. Fundamenta Informaticae 74 (2006) 301-328

12. Cimiano, P., Stumme, G., Hotho, A., Tane, J.: Conceptual knowledge processing with formal concept analysis and ontologies. In: Proc. 2nd Int. Conf. on Formal Concept Analysis (ICFCA 04). (2004) 189-207

13. Stumme, G., Hotho, A., Berendt, B.: Semantic Web mining: State of the art and future directions. Journal on Web Semantics 4 (2006) 124-143

14. Hitzler, P., Krötzsch, M.: Querying formal contexts with answer set programs. In Schärfe, H., Hitzler, P., Øhrstrøm, P., eds.: Proc. ICCS 2006. Volume 4068 of LNAI., Springer (2006) 413-426

15. Rudolph, S.: A deduction calculus for cumulated clauses on $\mathcal{F} \mathcal{L} \mathcal{E}$ concept descriptions. In Hitzler, P., Schärfe, H., Øhrstrøm, P., eds.: Inspiration and Application - Contributions to ICCS 2006, Aalborg University Press (2006) 188-201

16. Rudolph, S.: Exploring relational structures via $\mathcal{F} \mathcal{L} \mathcal{E}$. In Wolff, K.E., Pfeiffer, H.D., Delugach, H.S., eds.: Proc. ICCS 2004. Volume 3127 of LNCS., Springer (2004) 196-212

17. Tane, J., Cimiano, P., Hitzler, P.: Query-based multicontexts for knowledge base browsing: an evaluation. In Schärfe, H., Hitzler, P., Ohrstrom, P., eds.: Proc. ICCS 2006. Volume 4068 of LNAI., Heidelberg, Springer (2006) 